\title{
Das Palmengarten-Jahr 2011
}

\author{
Matthias Jenny
}

Wieder einmal liegt ein ereignisreiches Jahr mit spannenden Aktivitäten, Neuerungen und besonderen Blühereignissen hinter uns, an die wir uns gerne zurückerinnern. Deren Fülle ist allerdings so groß, dass die nachfolgende Zusammenstellung keinen Anspruch auf Vollständigkeit erheben kann.

Nach einer klassischen, frostigen und weißen Weihnacht 2010 startet der Januar mit Eis und viel Schnee, für viele Gewächse des Freilandes ein ziemlicher Stresstest. Doch im schönsten Garten Frankfurts können Wärmehungrige den lausigen Temperaturen ganz leicht entgehen und bei tropischem Klima in den Gewächshäusern unter Palmen wandeln. So auch die Vorschulkinder, die im Rahmen des neuen und erfolgreichen Projektes „Kinder im Garten“ zusammen mit ihren Kita-Gruppen in Dreitageskursen bestimmte Pflanzengruppen wie Bambus oder Palmen erkunden. Doch auch der härteste Winter hat ein Ende. Mitte Januar setzt starkes Tauwetter ein, der Main tritt im Innenstadtbereich heftig über die Ufer. Und schon strecken sich auf den Wiesen die ersten Schneeglöckchen und Krokusse der Sonne entgegen. Immer freudig erwartet wird die Kamelien-Ausstellung, die bereits im Winter eine Blütenpracht von Weiß bis Rot in die Ausstellung zaubert. Die Blumenausstellungen haben jedes Jahr ein anderes Motto. Diesmal ist die Ausstellung dem Leben und Schaffen HeinRICH Siesmayers gewidmet. Harmonisch in die Kamelien integriert ist eine von Barbara Vogt konzipierte Wanderausstellung über den bekannten Bockenheimer Kunstgärtner und Gründer unseres Palmengartens.

Wenn es draußen im Februar noch dunkel und kalt ist, ist der Palmengarten bereits dem Früh-

Abb. 1: Bunte Krokuswiese vor dem Tropicarium. ling voraus. Vom 18. Februar bis 6. März lockt die Galerie Ost mit 65000 Frühlingsblüten in die Frühlingsblumenausstellung. Diese Ausstellung ist immer ein Erlebnis für Auge und Nase, denn ein intensiver Duft von Hyazinthen und Narzissen erfüllt die Galerie. Ein Birkenwäldchen auf einer großformatigen Fotowand, blühende Ziermandeln und diverse andere Frühblüher machen so richtig Lust auf Frühling. Als Ergänzung zu den echten Blumen gibt es Blütenbilder der Künstler Bettina Richter und Udo Becker zu betrachten. Der Palmengarten ist nicht nur bei den Frankfurtern sehr beliebt. Nach einer Umfrage gehört er auch bei auswärtigen Besuchern zu den beliebtesten touristischen Zielen der Stadt.

Ein Höhepunkt der Palmengarten-Aktivitäten steht im März mit der Verkaufsausstellung Garten 2011 auf dem Programm. Vom 17. bis 20. März kommen Liebhaber von Pflanzenraritäten auf ihre Kosten, können diverses Gartenzubehör erwerben, und im Zelt von BioFrankfurt gibt es reichlich Informationen aus dem grünen Bereich. Die Verkaufsausstellung mit Raritätenbörse zieht 30000 Besucherinnen und Besucher von Nah und Fern an. Passend zur

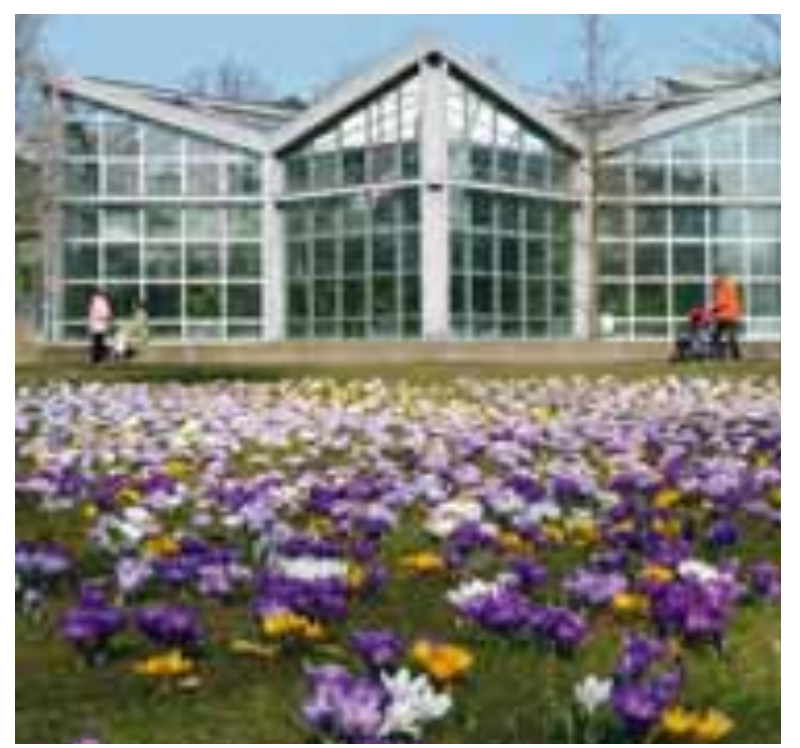



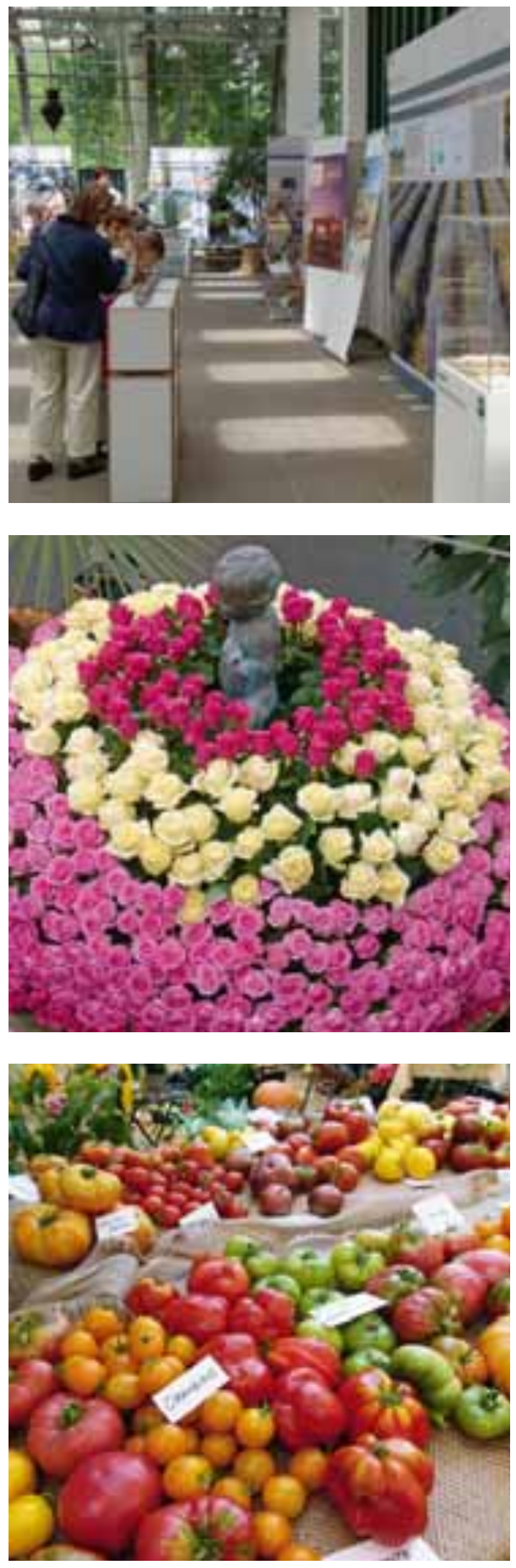

Ausstellungszeit schmückt sich der Palmengarten mit seinen Krokuswiesen, die in diesem März besonders bunt und prall blühen. Damit der Palmengarten immer wieder im neuen Glanz erscheint, müssen zahlreiche fleißige Hände mit anpacken. In Zukunft wird der Palmengarten noch größer sein, denn es steht nun fest, dass der Botanische Garten ab dem 1. Januar 2012 mit dem Palmengarten vereinigt und damit gerettet sein wird.

Ganz exotisch geht es auch im April zu. In der Orchideen-Ausstellung kann man in allen Farben schwelgen, diverse Infos über die Kultur von Orchideen bekommen und natürlich auch die Königin der Blumen für zu Hause erstehen. Mitte April zeigt der Palmengarten die schönsten Exemplare seiner Azaleen-Sammlung. Passend zur asiatischen Herkunft vieler Azaleen bereichern Bonsais die Ausstellung, darunter eine 800jährige Eibe („Baum des kaiserlichen Anblickes"). 2011 fällt Ostern so spät wie selten. Viele Osterglocken sind deshalb bereits verblüht, dafür blühen die ersten Pfingstrosen. Wie immer vor allem bei den Kleinen sehr beliebt ist das Osterhasengärtchen, das über die Feiertage in der Azaleen-Ausstellung aufgebaut wurde.

Der Frühling gibt sich heiß und viel zu trocken, wochenlang fällt kaum ein Tropfen Regen. Auf Hochtouren muss gewässert werden. Noch deutlich vor Sommerbeginn sehen einige Rasenflächen stellenweise braun und vertrocknet aus. Passend zu dem sommerlichen Ambiente zeigen wir vom 21. April bis 22. Mai die „Coco de mer und andere Schätze der Seychellen“. Botanisches rund um den größten Samen im Pflanzenreich und seine Darstellung in der regionalen Kunst und Fotografie gehen hier eine gelungene Synthese ein. Auch die lange Nacht der Museen steht im Zeichen der Seychellen. In einer lauen Nacht darf in der Galerie West zu heißen tropischen Rhythmen zusammen mit

Abb. 2 (oben): In der Ausstellung „Tausend und ein Öl“.

Abb. 3 (Mitte): Rosendekoration im Eingangsschauhaus.

Abb. 4 (unten): Tomatenvielfalt in der Erntedankausstellung. 
einer Tanzgruppe von den Seychellen getanzt werden. Im Rahmen dieser Ausstellung erhält der Palmengarten auch zwei keimfähige Seychellennüsse, um seinen Palmenbestand zu bereichern. Schon bald wird eine der beiden Nüsse keimen und im Tieflandregenwald, gut geschützt von einem kleinen Kunststoffhäuschen und gewärmt durch Fußbodenheizung, ausgepflanzt.

Ab Mai dreht sich dann alles um pflanzliche Öle und Fette. Die diesjährige große Informationsausstellung „Tausend und ein Öl“ erscheint in neuem Design mit einem verbesserten Ausstellungssystem. Auf den großen Ausstellungswänden kommen die großformatigen Fotos sehr gut zur Geltung. Auf das Thema stimmt im Eingangsbereich ein alter knorriger Ölbaum ein, der extra für die Ausstellung gekauft wurde. Es gibt viele Fettnäpfchen und Öltöpfchen zu sehen. Ein sehr hochwertiges Öl ist das Arganöl, das aus den Samen des in Marokko heimischen Arganbaumes gewonnen wird. Deshalb wurde Marokko als Gastland für die Ausstellung gewählt. Die abendliche Eröffnungsfeier fällt marokkanisch aus. Und an verschiedenen Stellen der Ausstellung erscheint das Thema Marokko, ob es nun diverse Produkte des Arganbaumes sind, marokkanisches Geschirr oder Rosenöl. Auch eine marokkanische Woche im Juli ergänzt das umfangreiche Rahmenprogramm. Am 10. Mai wird auch endlich der neue Wasserspielplatz eröffnet. Der alte Wasserspielplatz mit seiner von Enten regelmäßig besuchten freien Wasserfläche entsprach nicht mehr den aktuellen technischen und hygienischen Normen. Die Düsen geben jetzt Trinkwasser ab, wenn Kinder draufdrücken oder daran rumwackeln. Von dort aus nutzt der Palmengarten das Wasser zur Bewässerung der Rasenflächen. Dieses Projekt konnte dank großzügiger Unterstützung der Organisation „Ein Herz für Kinder“ und der Stadt Eschborn realisiert werden.

Der Juni ist Rosenzeit. Dazu gehört nicht nur der üppig blühende Rosengarten, sondern das traditionelle Rosen- und Lichterfest zusammen mit der Rosenausstellung. Zehntausende lockt es dann in den Garten. Ausnahmsweise dürfen die Rasenflächen betreten und belagert werden, um am Abend auf den Wiesen kreative Bilder aus unzähligen Teelichtern zu legen und mit Einbruch der Dunkelheit das 15-minütige imposante Feuerwerk zu bestaunen. Überall wächst und gedeiht es. Das neue Asienbeet neben dem Caféhaus Siesmayer und die Staudenpflanzung seitlich des Rosengartens entwickeln sich prächtig. Neue kleine Beete mit Pflanzen der Südhalbkugel sind vor dem Subantarktishaus und vor den Alpinhäusern entstanden. Auch die heranwachsende Familie Schwan mit ihren drei Jungen, zwei grauen und einem weißen, zieht immer wieder Aufmerksamkeit auf sich. Mit der Renovierung des Gesellschaftshauses geht es in kleinen Schritten zurück zur Pracht. Unvorhergesehene Schwierigkeiten wie z. B. der Befall des Gebäudes mit dem Hausschwamm, verzögern und verteuern jedoch die Fertigstellung. Geduld und ein paar BaustellenUnannehmlichkeiten sind angesagt, doch dann wird Frankfurt wieder seinen schönsten Festsaal haben.

Ab Juli ist es leider vorbei mit dem schönen Wetter. Der erhoffte Regen kommt, manchmal zum Leidwesen vieler Menschen in zu geballter Ladung. Unter dem verregneten und kühlen Sommer leiden auch viele Freiluftveranstaltungen. Immer wieder bangen die Organisatoren unserer zahlreichen sommerlichen Musikveranstaltungen darum, ob es trocken bleibt. Kammeroper mit der Zauberflöte, Weltmusik, Jazz im Palmengarten, Blues und Promenadenkonzerte bieten für jeden Musikgeschmack etwas.

Durch Kooperationen mit anderen Institutionen kann der Horizont erweitert werden. An mehreren Terminen im August ist die Frankfurter Sternwarte zu Gast im Palmengarten. In den Sommerferien können am Oktogonbrunnen faszinierende Blicke auf die Sonne geworfen werden. Im Palmengarten wird gern gefeiert. So steht am 14. August das GWH-Fest auf dem Programm. Auf der Spielwiese gibt es Vieles für Groß und Klein zu entdecken, können 
Früchte und Samen erraten werden oder Jungpflänzchen in selbst bemalte Blumentöpfe gepflanzt werden. Besondere Attraktion ist der Waldkauz Adele am Stand der „Wildtierfreunde e.V.“, dem man von ganz nah in die schönen großen schwarzen Augen blicken kann. Je nach Jahreszeit sind die verschiedenen Themengärten im Palmengarten besonders schön entwickelt. Im Hochsommer beeindrucken u. a. die Steppenwiese mit ihrem Wildblumencharakter sowie der Sommerflor um das Tropicarium und das Historische Eingangsschauhaus. Beide Pflanzungen sind stets sehr beliebte Fotomotive für Hobby- und Profifotografen. Mit seiner Pflanzenvielfalt gehört der Palmengarten zu den Top10-Gärten weltweit. Dieses Ergebnis teilte Umweltdezernentin Manuela RottMANN auf dem diesjährigen Jahresempfang den 250 geladenen Gästen mit.

Im September geht die Freilandpflanzensaison so langsam ihrem Ende entgegen. Ein großes Aufflammen gibt es noch einmal mit der Blütezeit der Dahlien und der Erntedankschau sowie dem Herbstfest. Rot-Orange-Farben geben den Ton an. In der Erntedankausstellung, die diesmal dem Thema Tomate gewidmet ist, begeistern fast 100 verschiedene Tomatensorten. Die Vielfalt der Früchte ist groß und reicht von kirschklein bis paprikagroß, rund, birnen- oder herzförmig, gelb, rot, orange, grün, violett oder mehrfarbig marmoriert.

Im Oktober heißt es dann Abschied nehmen von langjährigen Freizeitgeräten. Der PalmenExpress tritt seine letzte Fahrt an. Die Parkeisenbahn entspricht nicht mehr dem technischen Standard. Durch die Verlegung des künftigen neuen Eingangs Palmengartenstraße entfällt die Wendeschleife am PapagenoTheater. Es muss deshalb ein neuer Zug her, der vorwärts und rückwärts fahren kann. Es ist geplant, für uns exklusiv ein Sondermodell anzufertigen, das einer verkleinerten Version der ersten Frankfurt-Offenbacher Straßenbahn entspricht. Mit dem Bau eines neuen Lokschuppens (eigentlich eines kleinen Kopfbahnhofs) wurde in der Nähe des Zauns an der Mi- quelallee begonnen. Der Bootsweiher wird neu verpachtet, weshalb auch neue Boote, darunter zur Hälfte Tretboote, angeschafft werden. Jedes Boot wird den Namen einer Blume tragen.

Die traditionelle Chrysanthemenausstellung im Oktober und November würdigt die Blumen des Herbstes. Chrysanthemen haben in China und Japan einen hohen Stellenwert. Passend dazu findet in Frankfurt im November die "Japan Week" statt. Auch im Palmengarten dreht sich vom 5. bis 12 . November alles rund um Japan. In der Galerie zeigen japanische Kunsthandwerker ihr Können, gibt es sportliche Vorführungen und können Besucher Origami-Figuren falten lernen. Im Siesmayersaal wird mehrfach eine japanische Teezeremonie durchgeführt. In diesem Jahr fällt die Herbstfärbung ganz besonders kontrastreich aus. Seit einigen Jahren ist es aber nicht der goldene Oktober, sondern eher der November, der durch knallige Laubfarben von Amberbaum, Ginkgo oder Scharlacheiche aufgewertet wird.

Der Dezember ist mild, kein Hauch von Winter ist zu spüren. Noch ahnt niemand, welche extreme Kältewelle Deutschland in einigen Wochen überrollen wird. In der Weihnachtsausstellung jedoch hat es schon geschneit. "Weihnachten steht vor der Tür" lautet das Thema. Von der Schreinerei des Palmengartens eigens für die Ausstellung angefertigte Hausfassaden mit weihnachtlich geschmückten Türen, Fenstern und Vorgärten prägen das Bild der Ausstellung. Im Blütenhaus stimmen eine verschneite Hütte und Skier zwischen Weihnachtssternen auf die kalte und dunkle Jahreszeit ein. Neue Herausforderungen für das kommende Jahr stehen vor der Tür, darunter sicherlich das Zusammenwachsen von Botanischem Garten und Palmengarten. Matthias Jenny und Manfred Wessel, Technischer Leiter des Botanischen Gartens, haben sich über viele Jahre hinweg sehr um die Rettung des Botanischen Gartens verdient gemacht. Für ihr großes und erfolgreiches Engagement wurde ihnen vom Verband der Botanischen Gärten die ZanderMedaille 2011 verliehen. 